\title{
Measurement Uncertainty Propagation in Transistor Model Parameters via Polynomial Chaos Expansion
}

\author{
Alessandra Petrocchi, Arun Kaintura, Gustavo Avolio, Member, IEEE, Domenico Spina, Member, \\ IEEE, Tom Dhaene Senior Member, IEEE, Antonio Raffo, Member, IEEE, and Dominique M. M.-P. \\ Schreurs, Fellow, IEEE
}

\begin{abstract}
We present an analysis of the propagation of measurement uncertainty in microwave transistor nonlinear models. As a case study, we focus on residual calibration uncertainty and its effect on modelled nonlinear capacitances extracted from small-signal microwave measurements. We evaluate the uncertainty by means of the Polynomial Chaos Expansion (PCE) method and compare the results with the NIST Microwave Uncertainty Framework, which enables both sensitivity and Monte-Carlo (MC) analyses for uncertainty quantification in microwave measurements. We demonstrate that, for the considered application, PCE provides results in agreement with classical MC simulations but with a significant reduction of the computational effort.
\end{abstract}

Index Terms - Microwave measurements uncertainty, FET, nonlinear modeling, polynomial chaos expansion.

\section{INTRODUCTION}

$\mathrm{I}_{\mathrm{b}}^{\mathrm{N}}$ $\mathrm{N}$ the last decade the study of uncertainty in measurement based microwave transistor nonlinear models has increased significantly. In general, measurements are affected by systematic errors, random errors, and drift errors due to the instrumentation non-idealities. For vector network analyzer (VNA) measurements, these errors cause mismatch, finite directivity and non-ideal frequency response. Hence, calibration is a crucial step in microwave measurement systems, because it allows one to correct for most of the systematic effects introduced by the measurement system itself. Calibration procedures require the measurements of well-known standards. However, these standards are not perfect due to, for instance, mechanical tolerances in the fabrication process. These imperfections add uncertainty in the calibration and, consequently, in the device-under-test (DUT) measurements. In this work, we evaluate how residual

Manuscript received January 4, 2017. Manuscript accepted February 23, 2017. A. Petrocchi, G. Avolio and D. M. M.-P. Schreurs are with ESAT-Division TELEMIC, KU Leuven, B-3001 Leuven, Belgium, email:

\{alessandra.petrocchi, gustavo.avolio, dominique.schreurs\}@kuleuven.be.

G. Avolio is supported by FWO Vlaanderen.

A. Kaintura, D. Spina and T. Dhaene are with the Department of Information Technology, Internet Based Communication Networks and Services (IBCN), Ghent University - iMinds, Technologiepark-Zwijnaarde 15, B-9052 Gent, Belgium, email: \{arun.kaintura, dspina, tom.dhaene\}@intec.ugent.be.

A. Raffo is with the Engineering Department, University of Ferrara, 44122 Ferrara, Italy, email: antonio.raffo@unife.it. uncertainties in calibration propagate to the parameters of a field-effect transistor (FET) model extracted from multi-bias scattering (S-) parameters. We performed uncertainty analysis with the PCE approach [1], [2]. Next, we compared the results obtained by the PCE method with those obtained by the NIST Microwave Uncertainty framework (MUF) [3]. The NIST MUF is a tool for uncertainty quantification in microwave measurements, which enables both sensitivity analysis and Monte-Carlo simulations. In our analysis, we included uncertainties in the standards of the thru-reflect-line (TRL) calibration. These uncertainties were provided by the foundry and refer to tolerances in the fabrication process of the lines. In Table I we report the nominal value and uncertainty of the used transmission lines. Next, we used the corrected transistor measurements to extract the parameters of a FET model and propagated uncertainty through the extraction procedure, as illustrated in Fig. 1. Finally, we propagated uncertainty in the model parameters to the simulated temporal voltages and currents of the transistor.

We show that, for the considered application, PCE approach provides results comparable to classical MC simulations but with reduced computational effort.

\section{UNCERTAINTY QUANTIFICATION}

In this Section, we describe the procedure for the extraction of the parameters of the capacitive part of the FET model. The resistive part of the FET model, i.e., the drain-source current generator and the gate Schottky junction model, are replaced with Look- Up-Tables (LUT) derived directly from DC measurements and are assumed to be without uncertainty.

\section{A. Identification of the capacitive model}

The device under test is a gallium-arsenide (GaAs) pHEMT $(0.15 \mu \mathrm{m} \times 200 \mu \mathrm{m})$. The extraction of the capacitive core is based on multi-bias S-parameter measurements at $\mathrm{f}_{0}=5 \mathrm{GHz}$ and over the bias range: $0 \mathrm{~V}<\mathrm{V}_{\mathrm{ds}}<9 \mathrm{~V}$ and $-1.5 \mathrm{~V}<\mathrm{V}_{\mathrm{gs}}<0$ $\mathrm{V}$. We adopted Angelov model [4] in order to describe $\mathrm{C}_{\mathrm{gs}}$ (1) and $\mathrm{C}_{\mathrm{gd}}(2)$ nonlinearities, while $\mathrm{C}_{\mathrm{ds}}$ is fixed and equal to 34.7 $\mathrm{fF}$.

$$
\begin{aligned}
C_{g s}= & C_{g s_{-} \min }+C_{g s 0}\left(1+\tanh \left(P_{10}+P_{11} V_{g s}+P_{111} V_{d s}\right)\right) . \\
& \cdot\left(1+\tanh \left(P_{20}+P_{21} V_{d s}\right)\right)
\end{aligned}
$$




$$
\begin{aligned}
C_{g d}= & C_{g d_{-} \min }+C_{g d 0}\left(1-P_{111}+\tanh \left(P_{30}-P_{31} V_{d s}+P_{1 c c} V_{d s} V_{g s}\right)\right) \cdot \\
& \cdot\left(1+\tanh \left(P_{40}+P_{41}\left(V_{g s}-V_{d s}\right)-P_{111} V_{d s}\right)+2 P_{111}\right)
\end{aligned}
$$

Firstly, the parasitic elements were extracted and deembedded. Then, we carried out an initial estimation of the model parameters, followed by a numerical optimization to obtain the best fitting between measured and simulated capacitances (Fig. 1). Within this step, we used the calibrated S-parameters without uncertainty and obtained the nominal values of the model parameters, which are reported in Table II.

TABLE I

TRL CALIBRATION STANDARDS WITH ASSOCIATED STANDARD UNCERTAINTY: L AND W ARE THE LENGTH AND WIDTH OF THE LINES, H IS THE SUBSTRATE THICKNESS, AND SIGMA IS THE METAL CONDUCTIVITY.

\begin{tabular}{ccccc}
\hline \hline & $\mathrm{L}(\mu \mathrm{m})$ & $\mathrm{W}(\mu \mathrm{m})$ & $\mathrm{H}(\mu \mathrm{m})$ & $\sigma(\mathrm{S})$ \\
\hline Thru & $570 \pm 1.42$ & $71 \pm 1.42$ & $100 \pm 2.5$ & $2.05 \mathrm{e} 7 \pm 0.10 \mathrm{e} 7$ \\
Line 1 & $2880 \pm 1.42$ & $71 \pm 1.42$ & $100 \pm 2.5$ & $2.05 \mathrm{e} 7 \pm 0.10 \mathrm{e} 7$ \\
Line 2 & $1490 \pm 1.42$ & $71 \pm 1.42$ & $100 \pm 2.5$ & $2.05 \mathrm{e} 7 \pm 0.10 \mathrm{e} 7$ \\
Line 3 & $980 \pm 1.42$ & $71 \pm 1.42$ & $100 \pm 2.5$ & $2.05 \mathrm{e} 7 \pm 0.10 \mathrm{e} 7$ \\
Short & $285 \pm 1.42$ & $71 \pm 1.42$ & $100 \pm 2.5$ & $2.05 \mathrm{e} 7 \pm 0.10 \mathrm{e} 7$ \\
\hline \hline
\end{tabular}

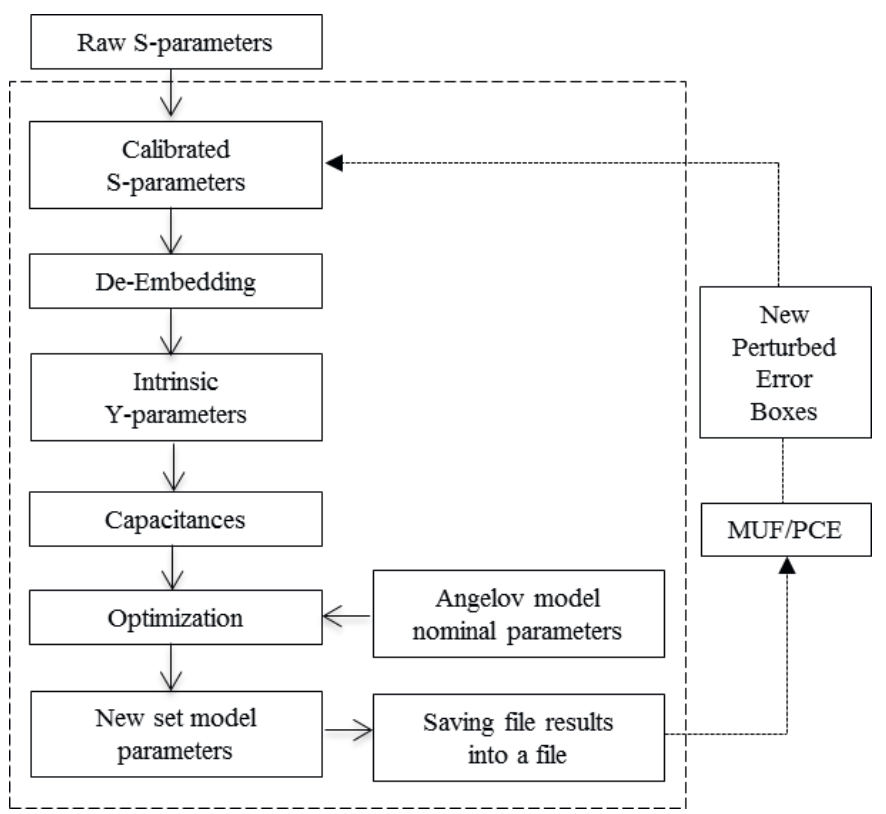

Fig. 1. Procedure for the identification of the capacitive-core model without the propagation of uncertainty (inside the dashed rectangle) and taking into account measurement uncertainty including MUF and PCE to generate perturbed error boxes.

\section{B. Uncertainty analysis with PCE method}

In the past years, PCE-based approaches were proposed in various engineering domains, such as in electronic and electromagnetic systems [5], [6]. However, to the authors' best knowledge, this is the first paper proposing the application of PCE to calibrate the uncertainty in measurement based transistor models, in order to reduce the computational time and resources required. The PCE model offers a strong mathematical rigorousness, giving an analytical representation of the statistical quantities of the system response: it is a stochastic expansion technique approximating a stochastic process by a series of orthogonal polynomials (also called basis functions) $\varphi_{i}(\xi)$ and suitable coefficients $\boldsymbol{\alpha}_{i}$. In the following, the variables $\xi$ are assumed independent, but PCE can be also applied in case of correlated ones. The PCE basis functions depend on the distribution of the input random variables $\boldsymbol{\xi}$ and, for specific distributions (i.e., Gaussian, Uniform, Beta), are the polynomials of the Wiener-Askey scheme [1].With the PCE, a stochastic process $\mathbf{Y}$ is expressed as:

$\mathbf{Y}=\sum_{i=0}^{\infty} \boldsymbol{\alpha}_{i} \varphi_{i}(\boldsymbol{\xi}) \cong \sum_{i=0}^{M} \boldsymbol{\alpha}_{i} \varphi_{i}(\boldsymbol{\xi})$

In practice, this infinite PCE series is truncated to a certain number of bases functions $M$ (a priori: depending on the maximum degree of the polynomials) such that the system response is accurately approximated [1], [5], [6].

Moreover, a recently proposed least angle regression selection (LARS) [2] approach allows computing a sparse PCE model: only those elements in (3) which have the largest influence on the PCE model response are selected.

In this Section, we consider the procedure described in Fig. 1. While in the literature simplified models have been used to accelerate optimization or parameter estimation [7], the aim of the proposed study is to quantify the uncertainty in the parameters of the capacitive core, as extracted from the Angelov model, by considering as sources of uncertainty fourteen independent Gaussian random variables related to the geometrical and physical characteristics of the calibration standards, as reported in Table I.

TABLE II

NOMINAL VALUE AND STANDARD UNCERTAINTY OBTAINED BY SENSITIVITY ANALYSIS (SA), MONTE CARLO (MC) ANALYSIS AND PCE METHOD

\begin{tabular}{lcccc}
\hline \hline Parameters & Nominal & SA & MC & PCE \\
\hline $\mathrm{C}_{\text {gs_min }}$ & $53.00 \mathrm{fF}$ & $0.87 \mathrm{fF}$ & $0.91 \mathrm{fF}$ & $0.90 \mathrm{fF}$ \\
$\mathrm{C}_{\mathrm{gs} 0}$ & $49.62 \mathrm{fF}$ & $0.94 \mathrm{fF}$ & $0.93 \mathrm{fF}$ & $0.92 \mathrm{fF}$ \\
$\mathrm{C}_{\text {gd_min }}$ & $25.24 \mathrm{fF}$ & $0.46 \mathrm{fF}$ & $0.44 \mathrm{fF}$ & $0.46 \mathrm{fF}$ \\
$\mathrm{C}_{\mathrm{gd} 0}$ & $28.22 \mathrm{fF}$ & $0.57 \mathrm{fF}$ & $0.56 \mathrm{fF}$ & $0.61 \mathrm{fF}$ \\
$\mathrm{P}_{40}$ & 4.001 & 0.006 & 0.005 & 0.005 \\
$\mathrm{P}_{11}=\mathrm{P}_{41}$ & 5.225 & 0.003 & 0.002 & 0.002 \\
$\mathrm{P}_{111}$ & 0.2315 & 0.0001 & 0.0001 & 0.0001 \\
$\mathrm{P}_{21}$ & 0.309 & 0.001 & 0.001 & 0.001 \\
$\mathrm{P}_{20}$ & -0.404 & 0.001 & 0.001 & 0.001 \\
$\mathrm{P}_{10}$ & 3.866 & 0.001 & 0.001 & 0.001 \\
$\mathrm{P}_{30}$ & 0.816 & 0.003 & 0.002 & 0.002 \\
$\mathrm{P}_{31}$ & 0.971 & 0.002 & 0.002 & 0.001 \\
$\mathrm{P}_{1 \mathrm{cc}}$ & -0.552 & 0.001 & 0.001 & 0.001 \\
\hline \hline
\end{tabular}

Thirty samples for the chosen random variables are generated by a Latin Hypercube Sampling technique and a corresponding Angelov model is built following the identification procedure described in Fig. 1. Next, sparse PCE models based on polynomials with maximum degree 2 are computed via LARS for each one of the Angelov parameters shown in Table II. Using LARS, for example in case of $\mathrm{C}_{\mathrm{gd} 0}$, results into only four number of terms in eq. 3, whereas a full PCE results into 120 elements. Note that stochastic moments can be obtained analytically from a PCE model [1], [2] and, if 
needed, more complex stochastic functions such as the probability density (PDF) or the cumulative distribution (CDF) functions [5], [6].

In Table II we report the standard deviation of the Angelov model parameters obtained analytically from the PCE models.

\section{RESULTS AND DISCUSSIONS}

In order to validate the proposed approach, we compared uncertainty obtained by PCE method to the sensitivity and MC analyses results obtained by the NIST MUF [8]. To this aim, we used the NIST MUF in combination with a commercial circuit simulator and we propagated the uncertainty through the model parameters extraction procedure as illustrated in Fig. 1. Standard uncertainty obtained by PCE and NIST MUF are in good agreement, as shown in Table II.

Furthermore, in order to determine the (global) sensitivity of the Angelov model parameters with respect to the chosen random variables, PCE based SOBOL analysis is carried out and compared with the sensitivity analysis results: for example, the SOBOL indices obtained for $\mathrm{C}_{\mathrm{gd} 0}$ by means of the PCE show a similar trend compared to the sensitivity analysis results reported in Fig. 2. The latter shows the contribution of the error mechanisms related to the calibration process. The highest contribution is given by substrate thickness and width for the line 1 which is also the longest line used in the calibration process.

Finally, we propagated uncertainty in the capacitive-core model to the simulated FET output temporal voltages and currents. PCE models are computed for the voltage and current at the transistor ports by means of the LARS algorithm, starting from the port signals values obtained for the 30 Angelov models computed so far.

In Fig. 3, the standard deviation of the extrinsic drain current obtained analytically by means of the corresponding PCE model is compared to the corresponding value obtained by means of the MC analysis, showing a good agreement between the two methods. Similar results can be obtained for the other port waveforms.

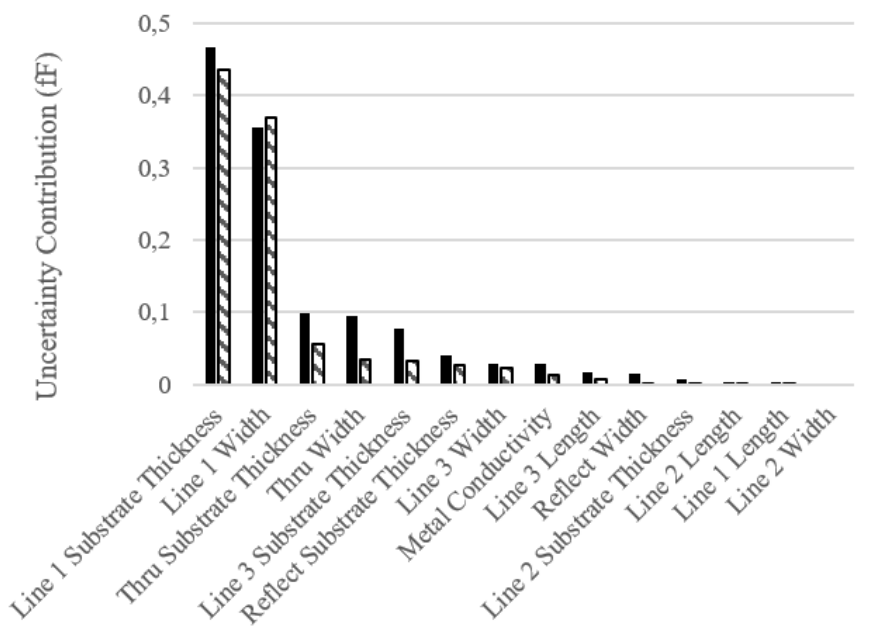

Fig. 2. Error contributions for PCE based SOBOL analysis (solid bars) and sensitivity analysis (diagonal filled bars).
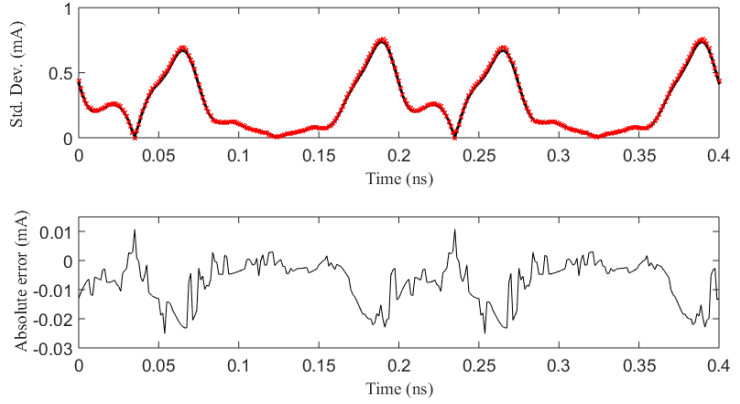

Fig. 3. Top: Standard deviation of the extrinsic drain current estimated by MC analysis (black continuous line) and PCE (red symbols). Bottom: Corresponding absolute error.

\section{CONCLUSIONS}

We propagated measurement uncertainty to a transistor model with a novel approach based on the PCE method. To validate the proposed approach, a comparison with the uncertainty analysis performed with the NIST Microwave Uncertainty Framework is shown. Specifically, we showed that the novel method provides results in good agreement with the classical Monte-Carlo method but with a significant reduction of the computational effort. Furthermore, PCE is able to achieve successful results with thirty simulations compared to the one hundred required for MC. It is also important to note that, for application with a major number of error mechanisms, one hundred MC will be not enough to get representative results. Increasing the number of $\mathrm{MC}$, it will require huge computational resources and it will be more time consuming.

The achieved results can contribute to give a new important insight to uncertainty quantification in measurement-based transistor models by considering the growing interest in uncertainty quantification in the field of microwave measurements.

\section{REFERENCES}

[1] D. Xiu and G. M. Karniadakis, "The Weiner-Askey Polynomial Chaos for Stochastic Differential Equations," SIAM J. Sci. Comput., vol. 24, no. 2, pp. 619-644, Apr. 2002.

[2] G. Blatman and B. Sudret, "Adaptive sparse polynomial chaos expansion based on least angle regression," J. Comput. Phys., vol. 230, no. 6, pp. 2345-2367, Mar. 2011.

[3] http://www.nist.gov/ctl/rf-technology/related-soft-ware.cfm.

[4] I. Angelov, H. Zirath, and N. Rorsman, "A New Empirical Nonlinear Model for HEMT and MESFET Devices," IEEE Trans. Microw. Theory Techn., vol. 40, no. 12, pp. 2258-2266, Dec. 1992.

[5] P. Manfredi and F. G. Canavero, "Efficient statistical simulation of microwave devices via stochastic testing-based circuit equivalents of nonlinear components," IEEE Trans. Microw. Theory Techn., vol. 63, no. 5, pp. 1502-1511, May 2015.

[6] D. Spina, F. Ferranti, T. Dhaene, L. Knockaert, G. Antonini, and D. Vande Ginste, "Variability analysis of multiport systems via polynomial-chaos expansion," IEEE Trans. Microw. Theory Techn., vol. 40, no. 8, pp. 2329-2338, Aug. 2012.

[7] F. Wang, S. Yin, T. Mukherjee, R. Negi, and L. Pileggi, "Re-thinking polynomial optimization: efficient programming of reconfigurable radio frequency (RF) systems by convexification," IEEE/ACM Asia and South Pacific Design Automation Conference, pp. 545-550, Jan. 2016.

[8] A. Lewandosky, D.F. Williams, P.D. Hale, J.C.M. Wang, and A. Dienstfrey, "Covariance-Matrix-Based Vector-Network Analyzer Uncertainty Analysis for Time- and Frequency-Domain Measurements," IEEE Trans. Microw. Theory Techn., vol. 58., no. 7, Jul. 2010. 